\title{
A Study on the Wettability of Ion-Implanted Stainless and Bearing Steels
}

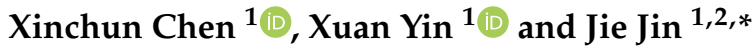 \\ 1 State Key Laboratory of Tribology, Tsinghua University, Beijing 100084, China; \\ chenxc1213@mail.tsinghua.edu.cn (X.C.); foebe@mail.tsinghua.edu.cn (X.Y.) \\ 2 School of Mechanical, Electronic and Control Engineering, Beijing Jiaotong University, Beijing 100044, China \\ * Correspondence: jjking200346@163.com; Tel.: +86-10-6278-9482
}

Received: 20 December 2018; Accepted: 8 February 2019; Published: 10 February 2019

\begin{abstract}
To satisfy the harsh service demand of stainless steel and aviation bearing steel, the anticorrosion and wettability behaviors of $9 \mathrm{Cr} 18$ stainless steel and M50 bearing steel tailored by ion beam surface modification technology were experimentally investigated. By controlling the ion implantation $\left(\mathrm{F}^{+}, \mathrm{N}^{+}, \mathrm{N}^{+}+\mathrm{Ti}^{+}\right)$or deposition processes, different surface-modified layers and ceramic layers or composite layers with both effects (ion implantation and deposition processes) were obtained on metal surfaces. The wettability was characterized by a contact angle instrument, and the thermodynamics stabilization of ion implantation-treated metals in corrosive solution was evaluated through an electrochemical technique. X-ray photoelectron spectroscopy (XPS) was employed for detecting the chemical bonding states of the implanted elements. The results indicated that ion implantation or deposition-induced surface-modified layers or coating layers could increase water contact angles, namely improving hydrophobicity as well as thermodynamic stabilization in corrosive medium. Meanwhile, wettability with lubricant oil was almost not changed. The implanted elements could induce the formation of new phases in the near-surface region of metals, and the wettability behaviors were closely related to the as-formed ceramic components and amorphous sublayer.
\end{abstract}

Keywords: ion implantation; M50 steel; wettability; DLC coating; contact angle

\section{Introduction}

Aeroengine bearings always work in complex conditions such as high temperature and load. Meanwhile, aeroengine bearings often encounter corrosive environments such as high humidity or salinity, as well as harsh environments during cross-sea flying or the berthing process [1-3]. Thus, it is urgently necessary to combat complex service environments through surface modification technologies to improve the corrosion and wear resistance of bearings under complex uses so as to extend service life. In practice, oil lubricant residing on the bearing surface can absorb a certain amount of water and corrosive $\mathrm{Cl}^{-}$. Therefore, surface modification treatments should improve the hydrophobicity of the steel materials without affecting the wettability of lubricants at the same time. Particularly, ion beam surface modification is a type of practical and convenient technology. Wettability control by ion beam surface modification is widely applied in hydrophobic material preparation for micro/nanoelectromechanical systems, improvements in biomaterial compatibility, anti-erosion, weldment, and lubrication of metal materials $[4,5]$. However, the wettability of certain materials sometimes cannot satisfy the necessary standards due to limitations in their chemical compositions and physical structure. Ion beam technology, especially ion implantation, is extensively utilized in research and applications for surface wettability through the modification of atomic configurations, chemical composition, and surface microstructure via high-energy ions, such as in the hydrophilic transformation of hydrophobic organic materials [6], the improvement of anticoagulation/calcification 
on biomaterial surfaces [7], and the reinforcement of welding performance [8,9]. Among these studies of wettability, most of them have only focused on organic materials and organic/metal combinations, and there have been few reports about ion implantation manipulation of wettability on pure metal surfaces. Although ion implantation can enhance anti-erosion [10] and tribological properties [11] of surfaces, wettability behaviors and underlying mechanisms in corrosive and lubricant mediums are still not clear after the ion implantation treatment.

The purpose of this paper was to utilize ion implantation/deposition technology to treat the surfaces of $9 \mathrm{Cr} 18$ stainless steels and M50 bearing steels through the implantation of various elements or film deposition plus implantation. M50 bearing steels are widely used in aeroengine bearings, and 9Cr18 stainless steels are even employed in the turbopump ball bearings of rocket engines [12,13]. Implanted elements such as $\mathrm{F}^{+}, \mathrm{N}^{+}$, and $\mathrm{N}^{+}+\mathrm{Ti}^{+}$and the deposition of some coatings, such as ceramic TiN $[14,15]$ and amorphous diamond-like carbon (DLC) [16], or a combination of these two methods, are thought to provide large potential for modifying the chemistry states of $9 \mathrm{Cr} 18$ and M50 steels. Meanwhile, contact angle tests of samples treated by ion implantation, deposition processes, or both were conducted on the basis of deionized water and aviation lubricating oil for different surface-treated samples, in order to find an appropriate ion beam surface modification technology that can endow bearing steels with hydrophobic corrosion resistance and wettability ability in aviation lubricating oil.

\section{Materials and Methods}

\subsection{Materials}

The experimental materials were 9Cr18 stainless steels (C: 0.90-1.00, $\mathrm{Si}: \leq 0.80, \mathrm{Mn}: \leq 0.80, \mathrm{~S}$ : $\leq 0.0 .30, \mathrm{P}: \leq 0.035, \mathrm{Cr}: 17.00-19.00, \mathrm{Ni}: \leq 0.60$, Mo: $\leq 0.75)$ and M50 bearing steels $(\mathrm{C}: 0.75-0.85, \mathrm{Si}:$ $\leq 0.35, \mathrm{Mn}: \leq 0.35, \mathrm{~S}: \leq 0.20, \mathrm{P}: \leq 0.27, \mathrm{Cr}: 3.75-4.25, \mathrm{Ni}: \leq 0.20$, Mo: 4.00-4.50), and their microstructures are shown in Figure 1. As seen from the morphologies, both steels are composed of martensite (needle-like or lath-shaped) and dispersive carbides (black dot-like), even though each individual phase is difficult to clearly distinguish. In addition, compared to stainless steel, M50 bearing steel samples have more homogeneous and miniaturized crystallites.
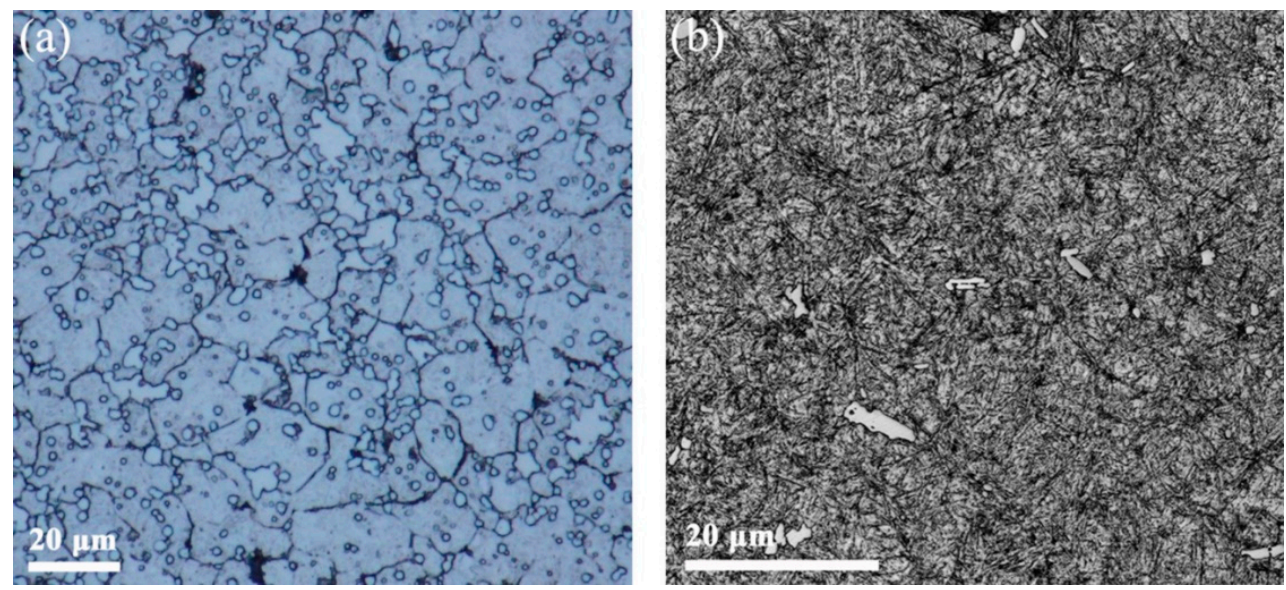

Figure 1. Metallurgical structures of (a) 9Cr18 stainless steel and (b) M50 bearing steel.

\subsection{Ion-Implanted Steels}

These samples were cut into spherical disks with a diameter of $20 \mathrm{~mm}$ and a thickness of $8 \mathrm{~mm}$ by wire-electrode cutting, and then polished step by step via abrasive paper, with $2000 \#$ as the last stage. Finally, they were polished by W2.5 diamond paste, yielding a surface roughness of 5-10 nm as measured by atomic force microscopy (AFM). Different doses of $\mathrm{F}^{+}, \mathrm{N}^{+}$, and $\mathrm{N}^{+}+\mathrm{Ti}^{+}$ions were implanted into the samples by an ion implanter and a MEVVA-source multifunctional coater (Hebei Riyueshan Sci \& Technol Corp, LZR-001, Hebei, China) to form modified sublayers with various 
compositions. The substrate was placed on the cooling stage, and the implantation process was periodically stopped for $2 \mathrm{~h}$ when an individual implantation dose of $0.5 \times 10^{17}$ ions $/ \mathrm{cm}^{2}$ was finished. Therefore, the total temperature increase of the substrate was below $50{ }^{\circ} \mathrm{C}$. The selected implantation energies were the maximum accelerating energies for the present ion implanter, with the purpose of inducing sufficient implantation depths. This choice was, on the one hand, to modify the wettability behaviors of steel surfaces, and on the other hand, to endow the modified surfaces with sufficient lubricating and wear-resistant implanted sublayers induced by ion implantation. Moreover, modified surfaces with diamond-like carbon (DLC) or a TiN coating synthesized by filtered vacuum cathodic arc, as well as a coating + ion implantation, were also prepared. Before deposition, the specimen surfaces were sputtered by $\mathrm{Ar}^{+}$to remove the contaminants. For DLC synthesis, a graphite (99.99\% purity) target was used as the source of carbon plasma for arc discharge. The resulting plasma beam was then passed through an off-plane $90^{\circ}$ bend solenoid to filter out particulates. The arc current was $55 \mathrm{~A}$, and the bias voltage applied to the substrate was set at $-100 \mathrm{~V}$. For TiN synthesis, a titanium target $(99.96 \%$ purity) and a working gas of $\mathrm{N}_{2}$ (99.99\% purity) were employed. The processed pressure was $0.2 \mathrm{~Pa}$. All the depositions were carried out at room temperature. The surface treatment parameters are listed in Table 1 . Note that the thickness of the DLC and TiN coating was $3 \mu \mathrm{m}$.

Table 1. Parameters of the as-treated specimens.

\begin{tabular}{|c|c|c|c|c|c|}
\hline Materials & $\begin{array}{l}\text { Sample } \\
\text { Number }\end{array}$ & $\begin{array}{l}\text { Implantation } \\
\text { Elements }\end{array}$ & $\begin{array}{c}\text { Implantation } \\
\text { Dose } / \times 10^{17} \text { Ions } / \mathrm{cm}^{2}\end{array}$ & $\begin{array}{l}\text { Implantation } \\
\text { Energy/keV }\end{array}$ & Coating \\
\hline \multirow{7}{*}{$\begin{array}{l}\text { 9Cr18 stainless } \\
\text { steel }\end{array}$} & SO & - & - & - & Textured \\
\hline & S1 & $\mathrm{F}^{+}$ & 0.5 & 400 & - \\
\hline & $\mathrm{S} 2$ & $\mathrm{~N}^{+}$ & 2 & 100 & - \\
\hline & S3 & - & - & - & DLC \\
\hline & S4 & $\mathrm{F}^{+}$ & 0.5 & 400 & DLC \\
\hline & S5 & - & - & - & $\mathrm{TiN}$ \\
\hline & S6 & $\mathrm{N}^{+}$ & 2 & 100 & $\mathrm{TiN}$ \\
\hline \multirow{3}{*}{$\begin{array}{l}\text { M50 bearing } \\
\text { steel }\end{array}$} & S7 & $\mathrm{N}^{+}+\mathrm{Ti}^{+}$ & $2\left(\mathrm{~N}^{+}\right)+2\left(\mathrm{Ti}^{+}\right)$ & $100\left(\mathrm{~N}^{+}\right)+90\left(\mathrm{Ti}^{+}\right)$ & - \\
\hline & M0 & - & - & - & - \\
\hline & M1 & $\mathrm{N}^{+}+\mathrm{Ti}^{+}$ & $2\left(\mathrm{~N}^{+}\right)+2\left(\mathrm{Ti}^{+}\right)$ & $100\left(\mathrm{~N}^{+}\right)+90\left(\mathrm{Ti}^{+}\right)$ & - \\
\hline
\end{tabular}

\subsection{Characterizations}

The metallurgical morphologies of samples were observed by an Olympus DSX500i (Olympus, Tokyo, Japan). The contact angles were recorded by an OCA20 video optical contact angle measuring instrument (Dataphysics, Filderstadt, Germany), which used deionized pure water and synthetic 4050 aviation lubricating oil as tested solution. Each liquid drop was $2 \mu \mathrm{L}$, and the measurement was finished within $2 \mathrm{~min}$. All the measurements were repeated at least 5 times to achieve the average value and to guarantee the reproducibility. The surface roughness was recorded by atomic force microscopy (AFM, Asylum Research MFP-3D, Oxford Instruments, Oxfordshire, UK). Micromorphologies were observed by high resolution transmission electron microscopy (HRTEM, JEOL 2010F, JEOL, Tokyo, Japan). Besides, the electrochemistry test was carried out by CHI660D (CH Instruments, Austin, TX, USA) under etchant solution with $5 \mathrm{wt} \% \mathrm{NaCl}$ dissolved in deionized water. The sample was first soaked in etchant solution for $20 \mathrm{~min}$, and then it was tested to record the stable potential values by open-circuit potential test. After the open-circuit potential was stable, the dynamic potential polarization curve was recorded from a positive orientation at a scanning speed of $1.67 \mathrm{mV} / \mathrm{s}$. The elemental and chemical states were measured by X-ray photoelectron spectrometer (XPS, Kratos, Manchester, UK) in an AXIS ULTRA DLD system, which utilized an Al target as a monochromated X-ray source and Al K $\alpha$ line energy at about $1486.6 \mathrm{eV}$.

\section{Results}

Figure 2 and Table 2 show the contact angles of 9Cr18 stainless steel samples with various treatments. In general, the water contact angle for a pure metal surface is between $60^{\circ}$ and $70^{\circ}$ due 
to its high surface energy. The relatively high contact angle of $88.9^{\circ}$ for $\mathrm{S} 0$ was due to the presence of textures fabricated on the sample surface. When $\mathrm{F}^{+}$and $\mathrm{N}^{+}$ions were implanted into S1 and S2 samples, respectively, their contact angles were slightly increased to be higher than $95^{\circ}$. However, after $\mathrm{F}^{+}$implantation, the contact angle was increased to $96.8^{\circ}(\mathrm{S} 4)$, which was mainly due to the hydrophobic nature of fluorine. When the TiN coating was deposited onto the surface of stainless steel (S5), the contact angle reached $102^{\circ}$. Further implantation by $\mathrm{N}^{+}$(S6) hardly changed the contact angle $\left(100.4^{\circ}\right)$. When $\mathrm{N}^{+}+\mathrm{Ti}^{+}$were co-implanted into steel (S7) to form a $\mathrm{TiN}_{x}$ nitride modified layer, the contact angle was improved to $93^{\circ}$.

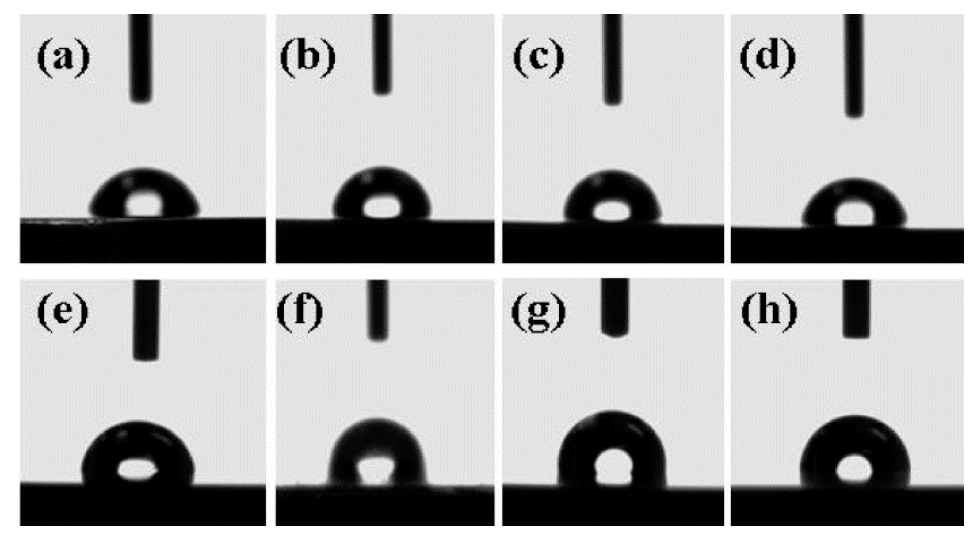

Figure 2. Optical images showing the contact angle measurements between deionized water and different surface-modified 9Cr18 stainless steel specimens: (a) S0, (b) S1, (c) S2, (d) S3, (e) S4, (f) S5, (g) S6, and (h) S7.

Table 2. The measured contact angles between different surface-modified 9Cr18 stainless steel specimens and deionized water.

\begin{tabular}{ccccccccc}
\hline Number & S0 & S1 & S2 & S3 & S4 & S5 & S6 & S7 \\
\hline Contact angle $\left(^{\circ}\right)$ & 88.9 & 98.8 & 96.3 & 90.5 & 96.8 & 102.1 & 100.4 & 93.3 \\
\hline
\end{tabular}

As shown in Figure 3, on the one hand, the amorphous DLC coating had a nanoscale smooth surface $\left(R_{\mathrm{a}}=12.5 \mathrm{~nm}\right)$, and on the other hand, the content of $s p^{3}-\mathrm{C}$ was over $65 \%$. The bonding fraction calculation followed the methodology proposed by Toro and Mezzi [17,18].

(a)

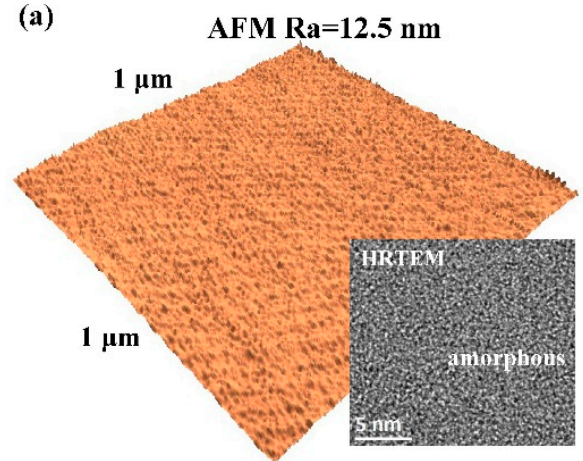

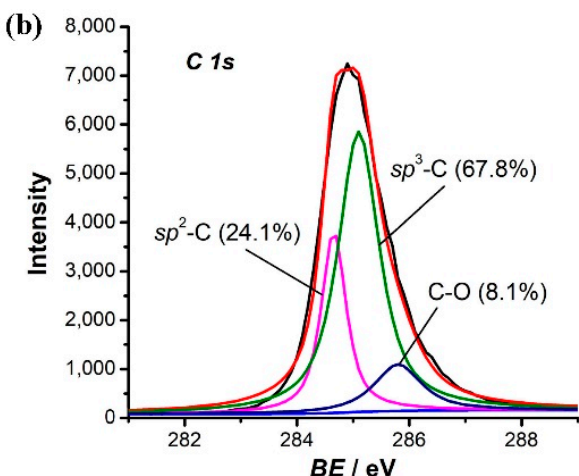

Figure 3. Structural characteristics of diamond-like carbon (DLC) coating (S3). (a) Nanoscale smooth surface indicated by AFM 3D morphology and an amorphous microstructure shown by an HRTEM image; (b) X-ray photoelectron spectrometer (XPS) fitting spectrum of $C 1$ s taken from the surface layer. BE: Binding energy.

Figure 4 shows the contact angles of M50 bearing steel samples before and after the ion implantation. The water contact angle of the pure sample was only $69^{\circ}$, and the contact angle 
of M50 bearing steel implanted by $\mathrm{N}^{+}+\mathrm{Ti}^{+}$ions (M1) was enhanced to be over $96^{\circ}$, which was similar to S7.

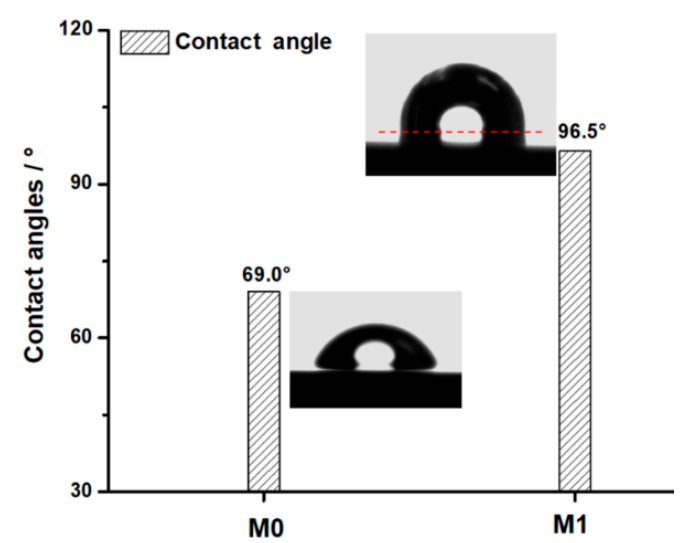

Figure 4. Evolution of contact angle measured by deionized water for bare (M0) and $\mathrm{N}^{+}+\mathrm{Ti}^{+}$ ion-implanted (M1) M50 bearing steel specimens.

As a comparison, S0, S1, S4, and M1 samples were chosen for lubricating oil contact angle evaluation, and the results are shown in Figure 5 and Table 3. Clearly, there were no obvious changes in the oil wettability behaviors before and after implantation or coating + implantation treatments to the sample surfaces. The contact angle was maintained in the region of $10^{\circ}-15^{\circ}$.

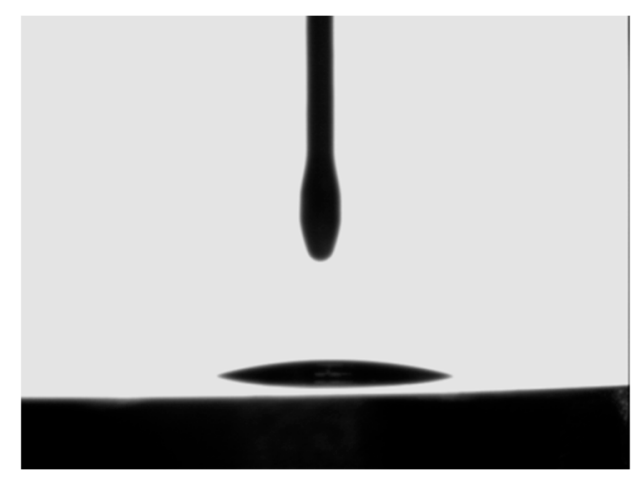

Figure 5. Optical image showing the contact angle measurement between the $9 \mathrm{Cr} 18$ stainless steel specimen S0 and lubricant oil.

Table 3. The measured contact angles between different 9Cr18 stainless steel and M50 bearing steel specimens and lubricant oil.

\begin{tabular}{ccccc}
\hline Number & S0 & S1 & S4 & M1 \\
\hline Contact angle $\left(^{\circ}\right)$ & 12.0 & 10.2 & 15.2 & 10.5 \\
\hline
\end{tabular}

The surface elemental composition and chemical binding states of the M1 sample were characterized by XPS after $\mathrm{N}^{+}+\mathrm{Ti}^{+}$implantation (Figure 6). It can be seen that the $\mathrm{N}^{+}+\mathrm{Ti}^{+}$-implanted surface was mainly composed of $\operatorname{TiN}_{x}, \mathrm{CrN}_{x}$, and titanium oxides. 
(a)

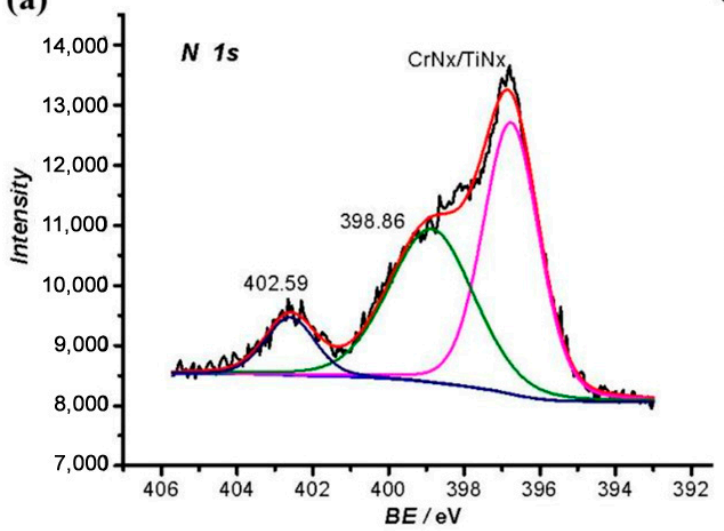

(b)

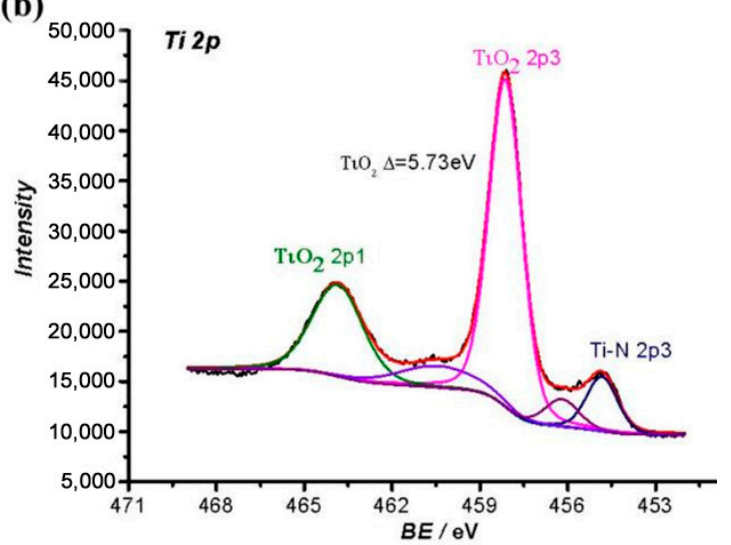

Figure 6. XPS spectra of (a) N1s and (b) Ti2p measured from the $\mathrm{N}^{+}+\mathrm{Ti}^{+}$-implanted M50 bearing steel specimen M1.

M50 bearing steel samples (M0 and M1, which had large changes in contact angle before and after ion implantation) were chosen to evaluate structural stability behavior in $5 \mathrm{wt} \% \mathrm{NaCl}$ corrosive medium through electrochemical measurements (Figure 7). The open circuit potentials before and after implantation were $-0.54 \mathrm{~V}$ and $-0.49 \mathrm{~V}$, respectively. The latter moved to a more positive position during the potential polarization test, where the corrosive current density decreased from 7.24 to $6.59 \mu \mathrm{A} / \mathrm{cm}^{2}$. The upper left corner of Figure 7 shows the slopes of the cathode branch and anode branch of the Tafel polarized area, achieved via CHI660D software fitting.

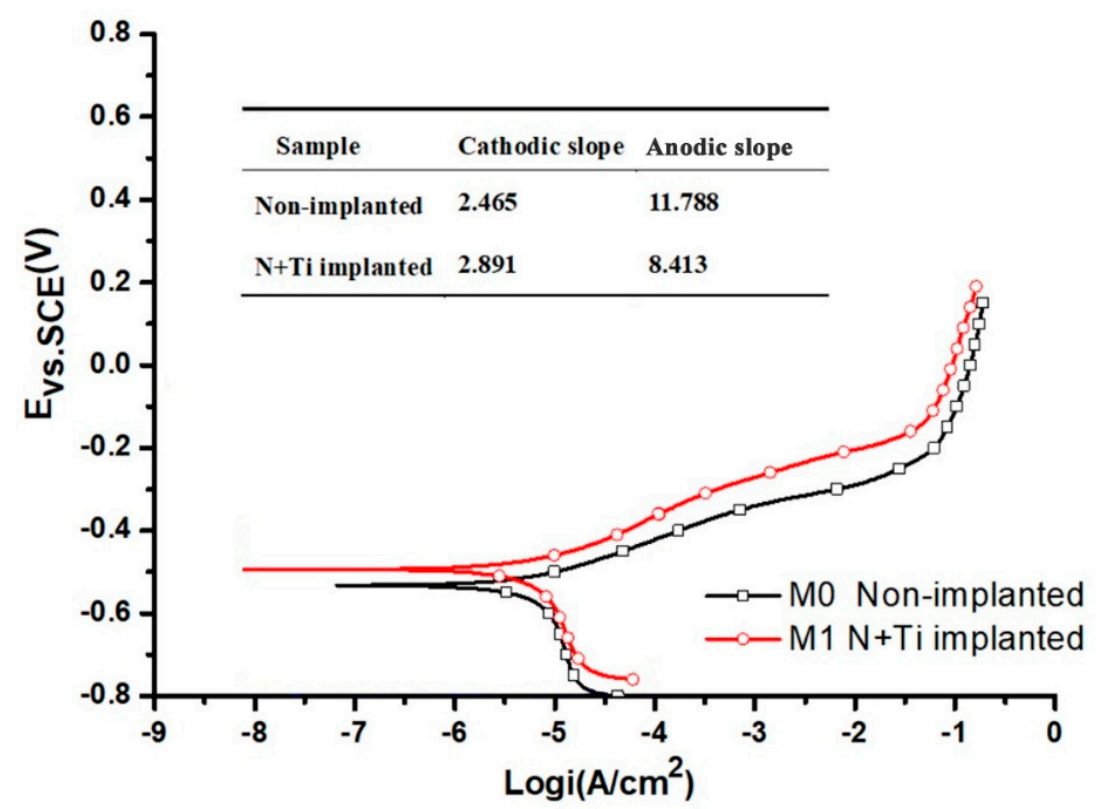

Figure 7. Potentiodynamic polarization curves measured for bare (M0) and $\mathrm{N}^{+}+\mathrm{Ti}^{+}$ion-implanted (M1) M50 bearing steel specimens.

\section{Discussion}

It is speculated that the presence of surface texture and a very thin oxide layer on a bare $9 \mathrm{Cr} 18$ stainless steel surface (as revealed by XPS) yielded a little bit larger of a contact angle $\left(88.9^{\circ}\right.$ ) for S0 (Figure 2 and Table 2). Interestingly, the contact angle of the $\mathrm{S} 1$ sample implanted by $\mathrm{F}^{+}$increased to $98.8^{\circ}$, mainly due to the hydrophobic nature of fluorine. In addition, note that the contact angle of the DLC coating on the S3 surface was comparable to the bare S0 sample, indicating the hydrophobic performance of DLC was almost similar to the textured stainless steel. However, after $\mathrm{F}^{+}$implantation 
into DLC, the contact angle increased to $96.8^{\circ}$ (S4), which was mostly consistent with the measured value of $97.3^{\circ}$ in a previous work [19]. When a TiN coating was deposited onto the surface of 9Cr18 steel (S5), the contact angle reached $102^{\circ}$. Further implantation by $\mathrm{N}^{+}$(S6) hardly changed the contact angle $\left(100.4^{\circ}\right)$. When $\mathrm{N}^{+}+\mathrm{Ti}^{+}$were co-implanted (S7) to form a $\mathrm{TiN}_{x}$ nitride modified layer, the contact angle improved to $93^{\circ}$. Comparing the aforementioned results, it can be seen that the water contact angles of steel surfaces were enhanced to some extent by $\mathrm{F}^{+}, \mathrm{N}^{+}$, and $\mathrm{N}^{+}+\mathrm{Ti}^{+}$implantation or TiN deposition, of which the formation of a ceramic TiN layer provided the best improvement. It should be pointed out that the water contact angle of the DLC coating in this work was $90.5^{\circ}$, higher than Kwok's work $\left(68.4^{\circ}\right)$ [20]. As shown in Figure 3, on the one hand, the amorphous DLC coating had a nanoscale smooth surface $\left(R_{\mathrm{a}}=12.5 \mathrm{~nm}\right)$, and on the other hand, the content of $s p^{3}-\mathrm{C}$ was over $65 \%$. Both of these effects endowed the DLC sample with relatively low surface energy, which was consistent with the values in a similar study [21]. The contact angle of DLC implanted by $\mathrm{F}^{+}$was further improved via forming an element-doped surface layer, and the increasing level was comparable to the $\mathrm{F}^{+}$-implanted 9Cr18. This indicates that $\mathrm{F}^{+}$implantation could tailor the hydrophobic properties via formation of a fluoride layer no matter whether it was on bare metal or a coated surface, while $\mathrm{N}^{+}$implantation could only influence the metal surface, rather than the ceramic TiN layer.

In Kwok's work, high surface energy P-DLC was synthesized by introducing phosphorus into a DLC matrix [20]. The more the chemical bonds changed, the more the surface energy increased. A completely hydrophilic transformation with a contact angle of about $16.9^{\circ}$ was realized in Kwok's work. Low surface energy organic materials generally have F- and Si-related bonds. Hence, the hydrophobic behavior induced by $\mathrm{F}^{+}$implantation in this work was probably associated with the change of chemical composition of the surface layer and crystal structure, which was also observed in a similar work [19]. However, the extent of F-induced hydrophobicity was not so significant as a result of the limited implantation dose available in the experiment.

The water contact angle of the pure M50 bearing steel sample was only $69^{\circ}$ (Figure 4 ), which was quite different from the water wettability behavior of the $9 \mathrm{Cr} 18$ stainless steel surface. This may have been related to the chemical structure of the surface passive oxide layer (Figure 6b). In addition, the contact angle of M50 bearing steel sample M1 was similar to S7. Obviously, co-implantation of $\mathrm{N}^{+}+\mathrm{Ti}^{+}$ could endow different steel surfaces (such as 9Cr18 stainless steel or M50 bearing steel) with similar interfacial water wettabilities, which implies that the hydrophilic characteristics of an ion implantation modified surface is mainly dependent on the species and dose of implanted elements rather than the structural nature of the substrates.

Clearly, there were no obvious changes in oil wettability behaviors before and after implantation or coating + implantation treatments to the 9Cr18 stainless steel surfaces (Figure 5 and Table 3). The contact angle was maintained in the region of $10^{\circ}-15^{\circ}$, indicating excellent oil wettability of the samples. Similarly, the results for M50 bearing steel materials also displayed no obvious changing of the interfacial behaviors after $\mathrm{N}^{+}+\mathrm{Ti}^{+}$implantation, indicating the well-maintained lipophilic properties of the samples.

The open-circuit potentials before and after implantation were $-0.54 \mathrm{~V}$ and $-0.49 \mathrm{~V}$, respectively (Figure 7). The latter moved to a more positive position, which indicated that the structural stability of M50 bearing steel in corrosive medium was improved by ion implantation treatment. During a potential polarization test, the corrosive current density decreased from 7.24 to $6.59 \mu \mathrm{A} / \mathrm{cm}^{2}$. The upper right corner of Figure 7 shows the slopes of the cathode branch and anode branch of the Tafel polarized area achieved via CHI660D software fitting. Therefore, ion implantation could effectively improve the corrosion resistance of metal materials, which was mainly related to its surface stability during the process of restraining the corrosion initiation point. From the perspective of microstructure, this was related to the ion implantation-induced formation of oxide and non-crystal layers on the surfaces. Combined with the results of contact angle measurements, ion implantation could change the wettability between the M50 surface and water. This variation could also significantly affect the structural stability of steel in a corrosive medium, which further suppressed the initiation of 
corrosion points. Moreover, the decrease in corrosive current density was associated with corrosion resistance phases formed inside the corroded sample, which tended to be bulk nature rather than interfacial behavior.

The results indicated that titanium oxide $\left(\mathrm{TiO}_{2}\right)$ and titanium nitride $\left(\mathrm{TiN}_{x}\right)$ were formed in the near-surface area after $\mathrm{N}^{+}+\mathrm{Ti}^{+}$implantation, which changed the structural characteristics of the metal materials' surfaces (Figure 6). Previous work has emphasized that high-energy ion implantation induced the formation of certain amorphous microcrystal structures or ceramic and oxide phases [22]. High-energy deposition not only induced the formation of a new phase as a working layer, but also caused a structural evolution inside the coating, such as the appearance of non-crystal and microcrystal phases [23]. Xiao demonstrated that the surface energy of materials decreased with decreasing grain size within an ultrafine grain size scope $(<20 \mathrm{~nm})$ through theoretical molecular dynamics [24]. Therefore, the wettability induced by $\mathrm{N}^{+}$and $\mathrm{N}^{+}+\mathrm{Ti}^{+}$high-energy ion implantation and high-energy ion coating of TiN was probably related to the formation of ceramic phases and an amorphous sublayer on the surface [25].

\section{Conclusions}

In summary, the wettability of metal materials' surfaces could be manipulated through a surface modification method such as ion implantation or deposition. The water contact angles of $9 \mathrm{Cr} 18$ stainless steel and M50 bearing steel increased to some extent through ion implantation or deposition of $\mathrm{F}^{+}, \mathrm{N}^{+}$, and $\mathrm{N}^{+}+\mathrm{Ti}^{+}$, indicating the suppression of wettability. In comparison, the DLC coating had no obvious influence on the water wettability of the substrate. The water contact angle of M50 bearing steel was remarkably enhanced after co-implantation of $\mathrm{N}^{+}+\mathrm{Ti}^{+}$, which delayed the trend of erosion in erosive medium. Moreover, ion implantation and coating had no obvious influence on the aero-oil contact angle for the two types of metal materials. In addition, the effect of ion implantation on the wettability of metal was mainly dependent on the new surface phases formation and bonding states change of the substrate. This technology is expected to become one effective anticorrosion method for aviation materials.

Author Contributions: X.C. conceived the project and discussed the results. J.J. performed the experiments and collected the data. X.Y. wrote the draft.

Funding: This research was funded by the National Natural Science Foundation of China under Grant No. 51605247 and the China Postdoctoral Science Foundation under Grant Nos. 2016M601008 and 2017T100069.

Conflicts of Interest: The authors declare no conflict of interest.

\section{References}

1. Shi, X.B.; Yan, W.; Xu, D.K.; Yan, M.C.; Yang, C.G.; Shan, Y.Y.; Yang, K. Microbial corrosion resistance of a novel Cu-bearing pipeline steel. J. Mater. Sci. Technol. 2018, 34, 2480-2491. [CrossRef]

2. Khan, F.A. The effect of soaking on segregation and primary-carbide dissolution in ingot-cast bearing steel. Metals 2018, 8, 800. [CrossRef]

3. Wu, H.X.; Ho, J.K.L.; Dong, G.N.; Zhang, D.Y. Friction reduction of pre-phosphating nanofilm on bearing steel by tricresyl phosphate pretreatment in boundary lubrication. J. Eng. Tribol. 2015, 229, 101-111. [CrossRef]

4. Smidt, F.A.; Hubler, G.K. Recent advances in ion beam modification of metals. Nucl. Instrum. Meth. B 1993, 80-81, 207-216. [CrossRef]

5. Cui, F.Z.; Luo, Z.S. Biomaterials modification by ion-beam processing. Surf. Coat. Technol. 1999, 112, $278-285$. [CrossRef]

6. Han, S.; Lee, Y.; Kim, H.; Kim, G.; Lee, J.; Yoon, J.; Kim, G. Polymer surface modification by plasma source ion implantation. Surf. Coat. Technol. 1997, 93, 261-264. [CrossRef]

7. Li, D.; Zhao, J.; Gu, H.; Lu, R.; Ding, F.; Zhang, Q. Effect of ion implantation on wettability, anticoagulability and anticalcification of polyurethane. Prog. Mater. Sci. 1993, 1, 66-99. [CrossRef]

8. Xu, M.; Li, Z.; Wang, G.; Wang, C.; Tian, X. Research on the microstructure and phase of interface of $\mathrm{Al}$ ion implanted stainless steel/Al joints. Rare Met. Mater. Eng. 2012, S2, 773-776. 
9. Dayss, E.; Leps, G.; Meinhardt, J. Surface modification for improved adhesion of a polymer-metal compound. Surf. Coat. Technol. 1999, 116-119, 986-990. [CrossRef]

10. Karimi, M.V.; Sinha, S.K.; Kothari, D.C.; Khanna, A.K.; Tyagi, A.K. Effect of ion implantation on corrosion resistance and high temperature oxidation resistance of Ti deposited 316 stainless steel. Surf. Coat. Technol. 2002, 158-159, 609-614. [CrossRef]

11. Li, X.; Yue, W.; Wang, C.; Gao, X.; Wang, S.; Liu, J. Comparing tribological behaviors of plasma nitrided and untreated bearing steel under lubrication with phosphor and sulfur-free organotungsten additive. Tribol. Int. 2012, 51, 47-53. [CrossRef]

12. Nosaka, M.; Oike, M.; Kikuchi, M.; Kamijo, K.; Tajiri, M. Tribo-characteristics of self-lubricating ball bearings for the LE-7 liquid hydrogen rocket-turbopump. Tribol. Trans. 1993, 36, 432-442. [CrossRef]

13. Gu, L.; Wang, L.Q.; Li, X.J.; Qi, Y.L. Study and review of liquid hydrogen/oxygen turbo-pump cryogenic bearing technology. Chin. Mech. Eng. 2002, 13, 86-89. [CrossRef]

14. Yazıcıa, M.; Kovacıb, H.; Yetimc, A.F.; Çelik, A. Structural, mechanical and tribological properties of Ti and TiN coatings on 316L stainless steel. Ceram. Int. 2018, 44, 14195-14201. [CrossRef]

15. Sun, T.; Wang, L.P.; Yu, Y.H.; Wang, Y.H.; Wang, X.F.; Tang, B.Y. TiN/ $\mathrm{ZrO}_{2}$ multilayers synthesized on GCr15 bearing steel using plasma immersion ion implantation and deposition. Surf. Coat. Technol. 2007, 201, 6615-6618. [CrossRef]

16. Azzi, M.; Paquette, M.; Szpunar, J.A.; Klemberg-Sapieha, J.E.; Martinu, L. Tribocorrosion behaviour of DLC-coated 316L stainless steel. Wear 2009, 267, 860-866. [CrossRef]

17. Mezzi, A.; Kaciulis, S. Surface investigation of carbon films: From diamond to graphite. Surf. Interface Anal. 2010, 42, 1082-1084. [CrossRef]

18. Toro, R.G.; Calandra, P.; Cortese, B.; de Caro, T.; Brucale, M.; Mezzi, A.; Federici, F.; Caschera, D. Argon and hydrogen plasma influence on the protective properties of diamond-like carbon films as barrier coating. Surf. Interfaces 2017, 6, 60-71. [CrossRef]

19. Yu, G.Q.; Tay, B.K.; Sun, Z. Fluorinated amorphous diamond-like carbon films deposited by plasma-enhanced chemical vapor deposition. Surf. Coat. Technol. 2005, 191, 236-241. [CrossRef]

20. Kwok, S.C.H.; Wang, J.; Chu, P.K. Surface energy, wettability, and blood compatibility phosphorus doped diamond-like carbon films. Diam. Relat. Mater. 2005, 14, 78-85. [CrossRef]

21. Moghadam, R.Z.; Ehsani, M.H.; Dizaji, H.R.; Kameli, P.; Jannesari, M. Modification of hydrophobicity properties of diamond like carbon films. Mater. Lett. 2018, 220, 301-304. [CrossRef]

22. Jin, J.; Chen, Y.; Gao, K.; Huang, X. The effect of ion implantation on tribology and hot rolling contact fatigue of Cr4Mo4Ni4V bearing steel. Appl. Surf. Sci. 2014, 305, 93-100. [CrossRef]

23. Jin, J.; Xu, Z.; Wang, J.; Ming, J.; Guo, Q.; Li, L. Property of multilayer TiCN films formed by ion-beam-assisted-deposition. Nucl. Technol. 2007, 30, 1019-1022. [CrossRef]

24. Xiao, D.; Xia, D.J. Effect of ultra-attenuation in surface energy decreasing. Therm. Sci. Technol. 2006, 1, 81-84. [CrossRef]

25. Jin, J.; Shao, T.M. Graded microstructure and mechanical performance of Ti/N-implanted M50 steel with polyenergy. Materials 2017, 10, 1204. [CrossRef]

(C) 2019 by the authors. Licensee MDPI, Basel, Switzerland. This article is an open access article distributed under the terms and conditions of the Creative Commons Attribution (CC BY) license (http://creativecommons.org/licenses/by/4.0/). 